\title{
The second-generation eCLIPs Endovascular Clip System: initial experience
}

\author{
${ }^{*}$ Albert H. Chiu, MBBS, FRANZCR,,${ }^{1-3}$ Joost De Vries, MD, PhD, ${ }^{4}$ Cian J. O’Kelly, MD, MSc, FRCSC, ${ }^{5}$ \\ Howard Riina, MD, ${ }^{6}$ lan McDougall, BASc, ${ }^{7}$ Jonathan Tippett, BASc, ${ }^{7}$ Martina Wan, PEng, BASc, ${ }^{7}$ \\ Airton Leonardo de Oliveira Manoel, MD, ${ }^{8}$ and Thomas R. Marotta, MD, FRCPC ${ }^{2,9}$
}

\begin{abstract}
${ }^{1}$ Neurovascular Program, ${ }^{3}$ Division of Neurosurgery, ${ }^{8}$ Keenan Research Centre, Li Ka Shing Knowledge Institute, and ${ }^{9}$ Diagnostic and Therapeutic Neuroradiology, St. Michael's Hospital, University of Toronto; ${ }^{2}$ Department of Medical Imaging, University of Toronto, Ontario; ${ }^{5}$ Division of Neurosurgery, University of Alberta, Calgary, Alberta; and ${ }^{7}$ Evasc Medical Systems Corporation, Vancouver, British Columbia, Canada; ${ }^{4}$ Radboud University Nijmegen, The Netherlands; and ${ }^{6}$ Department of Neurosurgery, NYU School of Medicine, NYU Langone Medical Center, New York, New York
\end{abstract}

OBJECTIVE Treatment of wide-necked intracranial aneurysms is associated with higher recanalization and complication rates; however, the most commonly used methods are not specifically designed to work in bifurcation lesions. To address these issues, the authors describe the evolution in the design and use of the eCLIPs (Endovascular Clip System) device, a novel hybrid stent-like assist device with flow diverter properties that was first described in 2008.

METHODS A registry was established covering 13 international centers at which patients were treated with the secondgeneration eCLIPs device. Aneurysm morphology and rupture status, device neck coverage, coil retention, and procedural and late morbidity and mortality were recorded. For those patients who had undergone successful implantation more than 6 months earlier, the final imaging and clinical follow-up results and need for re-treatment were recorded.

RESULTS Thirty-three patients were treated between June 2013 and September 2015. Twenty-five (76\%) patients had successful placement of an eCLIPs device; 23 (92\%) of these 25 patients had complete data. Eight cases of nondeployment occurred during the 1 st year of use, consistent with a learning curve; no failures of deployment occurred thereafter. Two periprocedural transient ischemic attacks and 2 asymptomatic thrombotic events occurred. Twenty-one (91\%) of 23 patients underwent follow-up at an average of 8 months (range 3-18 months); 9 (42.9\%) of these 21 patients demonstrated an improvement in Raymond grade at follow-up; no cases of worsening Raymond grade were recorded, and 17 (81.0\%) patients sustained a modified Raymond-Roy Classification class of I or II angiographic result at follow-up. Two delayed ruptures were recorded, both in previously coiled, symptomatic giant aneurysms where the device was used as a part of a salvage strategy.

CONCLUSIONS The second-generation eCLIPs device is a viable treatment option for bifurcation aneurysms. The aneurysm occlusion rates in this initial clinical series are comparable to the initial experience with other bifurcation support devices.

https://thejns.org/doi/abs/10.3171/2016.10.JNS161731

KEY WORDS interventional neuroradiology; intracranial aneurysm; embolization; therapeutic; flow diversion; radiology; bifurcation aneurysm; vascular disorders; interventional neurosurgery

$\mathrm{T}$ REATMENT of wide-necked bifurcation aneurysms is associated with higher recanalization and complication rates. ${ }^{5,7}$ Developments in the field of neurointervention have yielded various options for endovascular treatment of these lesions, including balloon-assisted coiling, stent-assisted coiling, and flow-diverter placement.
Although recent evidence suggests a safety and efficacy profile similar to that of standard coiling, ${ }^{8,13,16}$ most of these off-label techniques are not specifically designed to work in bifurcation lesions because, when positioning a balloon or stent across the neck of a bifurcation aneurysm into one daughter branch, generally only partial neck coverage

ABBREVIATIONS ICA = internal carotid artery; MCA = middle cerebral artery; MRRC = modified Raymond-Roy Classification; $P C A=$ posterior cerebral artery; WEB = Woven EndoBridge Aneurysm Embolization System.

SUBMITTED June 30, 2016. ACCEPTED October 13, 2016.

INCLUDE WHEN CITING Published online March 17, 2017; DOI: 10.3171/2016.10.JNS161731.

* Drs. Chiu and Marotta contributed equally to this work. 
can be achieved. With Y-stenting techniques, more secure neck protection can be achieved, but the technique is more time consuming and technically challenging. To address these issues, we describe the design and use of the secondgeneration eCLIPs device (Fig. 1; Evasc Medical Systems Corp.), an evolution in the novel flow-diverting stent-like assist device, which was first described in 2008. ${ }^{10}$

\section{Methods}

\section{The eCLIPs Device}

The eCLIPs device is a self-expanding, nickel-titanium, implantable noncircumferential device with platinum radiopaque markers. The device has 2 discrete sections: an anchor segment designed to conform to the arterial walls beside the neck of a bifurcation aneurysm, and a leaf segment with moveable ribs, designed to allow a coil delivery catheter to pass through the implanted device so as to deliver detachable coils. The leaf segment is designed to divert blood flow and reduce the jet effect into the aneurysm, thus reducing the chances of aneurysm recurrence, in a manner similar to current flow-diverter devices. The greater density of the leaf segment of the eCLIPs device also provides a scaffold for progressive neointimal growth over the aneurysm neck. Studies in animal models conducted prior to use in humans have demonstrated this effect, which undoubtedly increases the likelihood of complete aneurysm closure (Fig. 2). ${ }^{11}$

The second-generation device differs from the firstgeneration device ${ }^{10}$ in that it is not balloon mounted, it comes in a single size suitable for parent vessels ranging from 2.0 to $3.25 \mathrm{~mm}$ in diameter, and it does not circumferentially cover any of the parent vessel. The redesigned device is a self-expanding, microcatheter-delivered, fully retrievable, and self-orientating device. It provides immediate flow disruption into the aneurysm and also allows the placement of detachable coils without prior jailing of catheters or branching vessels.

\section{Patient Population}

A de-identified cohort of patients with bifurcation aneurysms were treated with the eCLIPs device at 13 neurointerventional centers in North America and Europe between June 2013 and September 2015 inclusive. Research ethics board approval was obtained. Each case was reviewed by a multidisciplinary team. Patients were treated using one of 2 paradigms. An initial group of patients with particularly complex lesions were treated with the eCLIPs device due to their "no other therapeutic option" status (Canada's Special Access Programme). A second group of patients were treated after CE Mark status was obtained. Patients in Europe who met inclusion criteria contained in the eCLIPs "Instructions for Use" document and were deemed amenable to treatment with the eCLIPs device were presented with 2 options: conventional stentassisted coiling, or treatment with the second-generation eCLIPs device. Written informed consent was obtained. All surgeons performing the procedure were trained by the same proctor (T.R.M.). Data collected at the time of procedure included aneurysm morphology (dome width, neck length, dome/neck ratio), location, rupture status (yes/no), number implanted, complete neck bridging (yes/ no), improvement in contrast stasis immediately following implantation (yes/no), modified Raymond-Roy Classification (MRRC) class after treatment with coils, coil retention (yes/no), procedural stroke and death, and any other adverse events. ${ }^{12}$ The standard first follow-up occurred at 6 months, but it happened earlier if there were clinical concerns. Data collected at follow-up were assessed by the individual site and included duration to follow-up, type of imaging follow-up, occlusion score, device migration, vessel thrombosis/dissection, interval stroke, death, retreatment, or other clinical adverse event.

\section{Procedural Anticoagulation Regimen and Antiplatelet Regimen}

Procedures were performed under general anesthesia. Systemic heparinization was administered with general dosages in the range of 50-100 U/ kg to a target active clotting time of more than $300 \mathrm{U} / \mathrm{kg}$ or 2-3.5 times baseline, depending on the neurointerventionist's preference, and was not continued after the operation. Antiplatelet regimens varied among operators. For elective cases, patients received $325 \mathrm{mg}$ aspirin between 2 and 5 days prior to the procedure, including the day of surgery, and either $75 \mathrm{mg}$

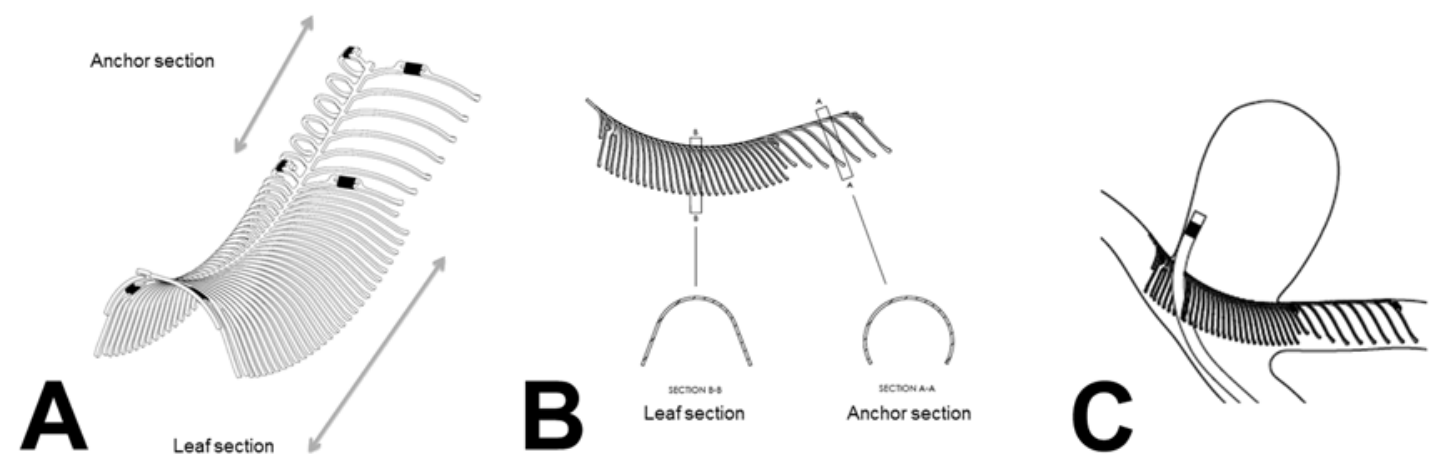

FIG. 1. A: Schematic of the eCLIPs device, demonstrating the leaf section, designed to lie over the neck of the aneurysm with increased metal coverage to promote flow diversion and coil support, and the anchor section, designed to lie on the vessel wall beside the neck. B: Schematic demonstrating the different cross-sectional profiles of the eCLIPs device. The leaf section is wider, maximizing neck coverage. C: En face schematic of a bifurcation aneurysm demonstrating the intended deployment position of the eCLIPs device, which does not jail either branch of the bifurcation, and allows microcatheter access to the aneurysmal sac. 


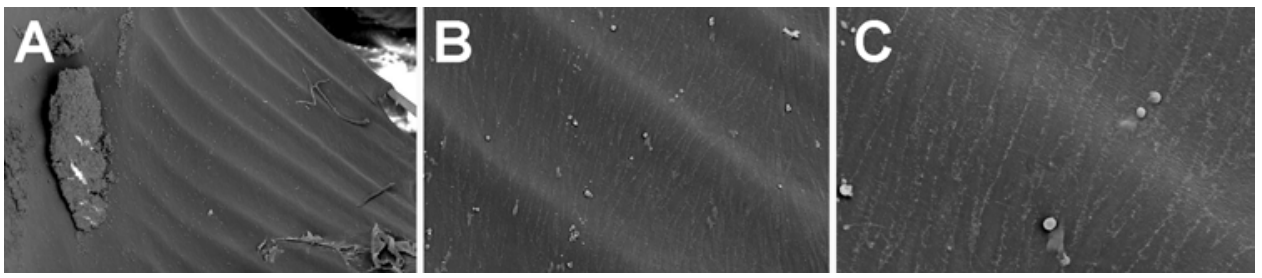

FIG. 2. Scanning electron microscopy images of eCLIPs explants from a model of carotid aneurysm bifurcation in rabbits at 90 days. Sequential magnifications of the eCLIPs device showing complete incorporation of the device ribs with well-organized, endothelialized neointimal overgrowth. Original magnification $\times 50(\mathbf{A}), \times 200(\mathrm{~B})$, and $\times 600$ (C).

clopidogrel daily or $10 \mathrm{mg}$ prasugrel daily 5 days prior to surgery (including the day of surgery), and continued receiving a dual antiplatelet blockade for a minimum of 3 months. If there were no complications or other concerns, the clopidogrel or prasugrel was stopped at 3 months and the patient continued on a regimen of aspirin monotherapy for at least 6 months. For acute cases, patients were given $650 \mathrm{mg}$ aspirin and either $600 \mathrm{mg}$ clopidogrel or $60 \mathrm{mg}$ prasugrel on the operative day. P2Y12 testing was not performed.

\section{Unique Delivery Method of the eCLIPs Device}

After guide catheter placement, the delivery system is assembled, consisting of placing a 0.014 -in microguidewire of choice through an eCLIPs micro-introducer; this is then threaded coaxially into an eCLIPs microcatheter. The system is advanced through the guide catheter into the intracranial circulation, past the target bifurcation aneurysm into one of the branch arteries over the 0.014-in guidewire. The guidewire and the eCLIPs micro-introducer are removed from the eCLIPs microcatheter, leaving the microcatheter in place in the branch artery (Video 1).

VIDEO 1. Video of a bifurcation aneurysm model demonstrating the sequential catheterization of the right daughter branch, deployment of the anchor section, wiring of the left daughter branch, and deployment of the leaf section followed by detachment of the device and collection of the delivery wire. Copyright Thomas Marotta.

Published with permission. Click here to view.

The existing guidewire or a new 0.014-in guidewire is then loaded into the eCLIPs Bifurcation Remodelling System, which delivers the eCLIPs device. The eCLIPs Bifurcation Remodelling System is pushed through the eCLIPs microcatheter until the eCLIPs device is at the end of the eCLIPs microcatheter. The device is partially deployed, and its axial and rotational orientations are evaluated. At this point, the device can be retracted within the eCLIPs microcatheter, repositioned, and partially redeployed.

Once the device is in the correct rotational and axial orientation, it is fully unsheathed in the first branch and partially into the parent artery. The guidewire is then pulled back and advanced into the second branch. The eCLIPs System is advanced over the guidewire, and the eCLIPs device is placed into the second branch. At any time throughout this procedure, the eCLIPs device can be fully recaptured for repositioning or replacement.

Once the eCLIPs device is in the correct orientation across the neck of the aneurysm, the eCLIPs detacher is used to detach the eCLIPs device from the delivery hypotube, thereby permanently implanting it in the artery. At this time the eCLIPs device can be crossed with a guidewire and any commercially available coil delivery microcatheter, facilitating placement of embolic coils behind the device.

\section{Results}

Data were collected for 33 patients at 13 international neurointerventional centers between June 2013 and September 2015. Twenty-five patients underwent successful implantation of an eCLIPs device; 21 aneurysms were basilar tip bifurcation aneurysms, 3 were terminal carotid artery aneurysms, and 1 was a middle cerebral artery (MCA) bifurcation aneurysm. Ten implants were placed in 9 patients in Canada under Health Canada's Special Access Programme between June 2013 and March 2015. Seventeen implants were placed in 16 patients in Europe; these placements were performed under individual application for use on compassionate grounds from September 2014 to January 2015. CE Mark status was gained in January 2015, and all cases in Europe until September 2015 were performed using CE Mark. From this cohort, 25 patients had reached their 6-month postprocedural followup; 23 (92\%) of these 25 patients had complete data at time of the procedure; complete late follow-up data were available in $21(91.3 \%)$ of the 23 patients. Aneurysm and follow-up data for the 25 patients with complete data are presented in Table 1. Eight (32\%) of 25 aneurysms had been previously treated with coils, and 1 had previous Woven EndoBridge Aneurysm Embolization System (WEB; Sequent Medical) treatment 2 years and 8 months earlier. Two (8\%) of the 25 patients were treated for acute rupture, and $5(20 \%)$ underwent treatment for aneurysms that had previously ruptured. Sixteen (64\%) of the 25 aneurysms were smaller than $10 \mathrm{~mm}$ in maximum diameter, and 2 (8\%) were giant $(\geq 25 \mathrm{~mm})$.

Technically successful deployment was achieved in 25 (76\%) of 33 cases. The device successfully retained coils in all cases in which it was attempted (Fig. 3). In 2 cases no coils were placed at the time of eCLIPs implantation, as the aneurysms were small and the flow-diverting effect was felt to be adequate in promoting subsequent thrombosis. At the end of treatment, 3 (13.0\%) of 23 patients had MRRC Class I occlusion, 8 (34.8\%) had Class II occlusion, 7 (30.4\%) had Class IIIa occlusion, and 5 patients (21.7\%) had Class IIIb occlusion. Two of 25 patients had no MRRC data available at the time of the procedure and therefore were not included in the follow-up analysis.

Nondeployment was recorded in 8 (75.8\%) of 33 cases. 
TABLE 1. Patients who underwent successful implantation of the eCLIPs device

\begin{tabular}{|c|c|c|c|c|c|}
\hline $\begin{array}{l}\text { Case } \\
\text { No. }\end{array}$ & $\begin{array}{l}\text { Aneurysm } \\
\text { Location }\end{array}$ & Previous Treatment & $\begin{array}{c}\text { MRRC Class Immediately } \\
\text { Posttreatment }\end{array}$ & $\begin{array}{l}\text { Longest Follow-Up } \\
\text { (mos) }\end{array}$ & $\begin{array}{c}\text { MRRC Class at } \\
\text { Longest Follow-Up }\end{array}$ \\
\hline 1 & Basilar tip & Coiled electively & Illb & 7 & IIlb \\
\hline 2 & Basilar tip & None & IIla & 18 & IIla \\
\hline 3 & MCA & None & Illb & ND & ND \\
\hline 4 & Basilar tip & None & II & 3 & II \\
\hline 5 & Basilar tip & None & Illa & 10 & II/IIla \\
\hline 6 & Basilar tip & Coiled $\times 2$ electively & Illb & 12 & Illb \\
\hline 7 & Basilar tip & None & II & 15 & I \\
\hline 8 & Basilar tip & Coiled for rupture & II & 9 & II \\
\hline 9 & Carotid terminus & None & II & 6 & I \\
\hline 10 & Basilar tip & Coiled for rupture & I & 6 & I \\
\hline 11 & Basilar tip & None & Illa & 5 & II \\
\hline 12 & Basilar tip & None & I & NA (unrelated death) & NA (unrelated death) \\
\hline 13 & Basilar tip & Coiled for rupture & II & 6 & II \\
\hline 14 & Carotid terminus & None & Illa & 6 & I \\
\hline 15 & Basilar tip & Coiled for rupture & II & 6 & II \\
\hline 16 & Basilar tip & None & I & 5 & I \\
\hline 17 & Basilar tip & WEB & Illb & 5 & I \\
\hline 18 & Basilar tip & Coiled electively & Illb & 6 & II \\
\hline 19 & Basilar tip & Coiled for rupture & II & 6 & II \\
\hline 20 & Basilar tip & None & II & 6 & II \\
\hline 21 & Basilar tip & None & Illa & 10 & II \\
\hline 22 & Carotid terminus & None & Illa & 10 & I \\
\hline 23 & Basilar tip & None & I & 10 & II \\
\hline 24 & Basilar tip & None & ND & ND & ND \\
\hline 25 & Basilar tip & None & ND & ND & ND \\
\hline
\end{tabular}

$\mathrm{NA}=$ not applicable $; \mathrm{ND}=$ no data.

The first was in a patient treated electively for an anterior communicating artery aneurysm. Initial attempted eCLIPs deployment and subsequent attempted stent-assisted coiling with a Solitaire AB device (Covidien-Medtronic) were both complicated by transient thrombosis formation, which resolved asymptomatically with device removal on both occasions without clinical complication. Dual-antiplatelet therapy resistance was suspected, although this was never formally tested, and the aneurysm was surgically clipped. The second patient had a wide-necked basilar
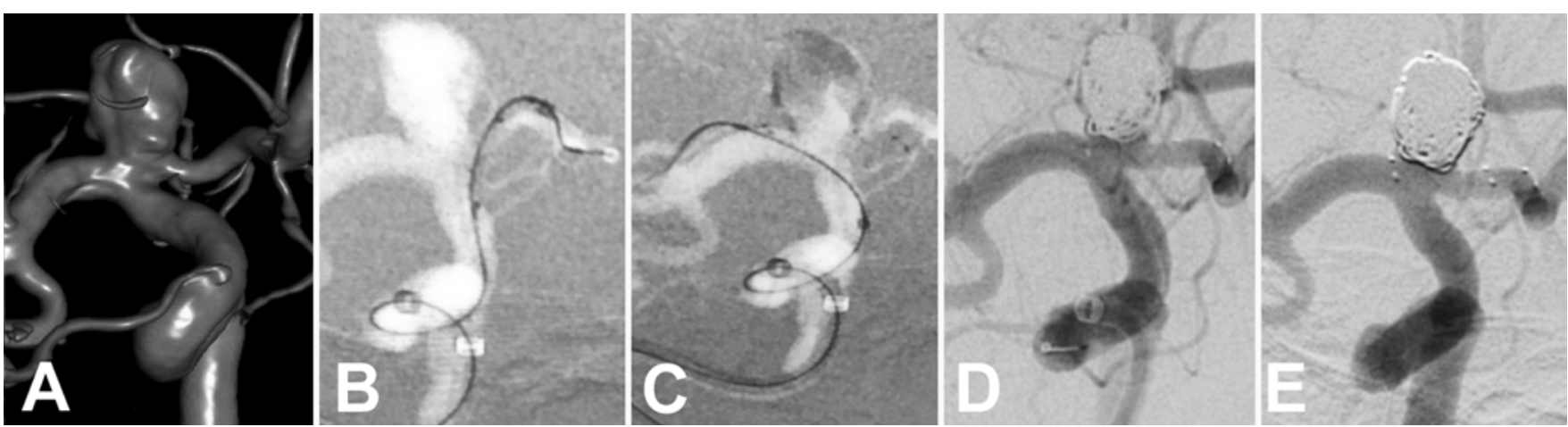

FIG. 3. A: Right ICA rotational angiogram (anteroposterior view) of a representative case treated with eCLIPs and coils, demonstrating an unruptured $10-\mathrm{mm}$ right ICA terminus aneurysm in a 64-year-old woman. B: Right ICA roadmap fluoroscopy image (frontal projection) demonstrating delivery of the eCLIPs device into the right $A_{1}$ segment of the anterior cerebral artery. C: Deployment of the eCLIPs device across the neck of the aneurysm with stasis of contrast within the aneurysmal dome, indicative of flow diversion effect. D: Right ICA angiogram (frontal projection) demonstrating the immediate postprocedural appearance with coiling of the aneurysmal dome post-eCLIPs placement. E: Right ICA angiogram (frontal projection) demonstrating the appearance at the 6-month follow-up with no evidence of neck recurrence. 
tip aneurysm, but posterior cerebral artery (PCA) spasm was encountered on delivering the microcatheter, and thus the patient underwent incomplete aneurysmal dome coil packing with the aid of balloon assistance; there were no associated complications (Fig. 4). The third patient had a recurrent unruptured aneurysm in the terminal segment of the internal carotid artery (ICA) that had undergone coiling 1 year previously. Satisfactory eCLIPs deployment across the neck could not be achieved because of vessel tortuosity, and the aneurysm with a small residual neck was repacked without an assisting device, and the patient awoke without complication. The aneurysm recurred again after 2 months and it underwent surgical clipping. The fourth patient had a fusiform right MCA bifurcation aneurysm for which double eCLIPs placement was planned, one into each limb; however, problems were encountered with device orientation and the procedure was abandoned. The fifth patient had a ruptured basilar sidewall blisterlike aneurysm. The eCLIPs device was chosen as a flow-diverting device; however, satisfactory rotational orientation could not be achieved, and the procedure was complicated by an embolus into the catheterized right PCA. This was retrieved via a stentriever thrombectomy, and the sidewall blister aneurysm was treated with a Pipeline Embolization Device (ev3, Inc.), resulting in closure of the aneurysm at 2 months. A sixth patient was treated for an unruptured left MCA ( $\mathrm{M}_{2}$ segment) aneurysm, but correct rotational alignment could not be obtained in part due to proximal tortuosity, and the patient was treated with stent-assisted

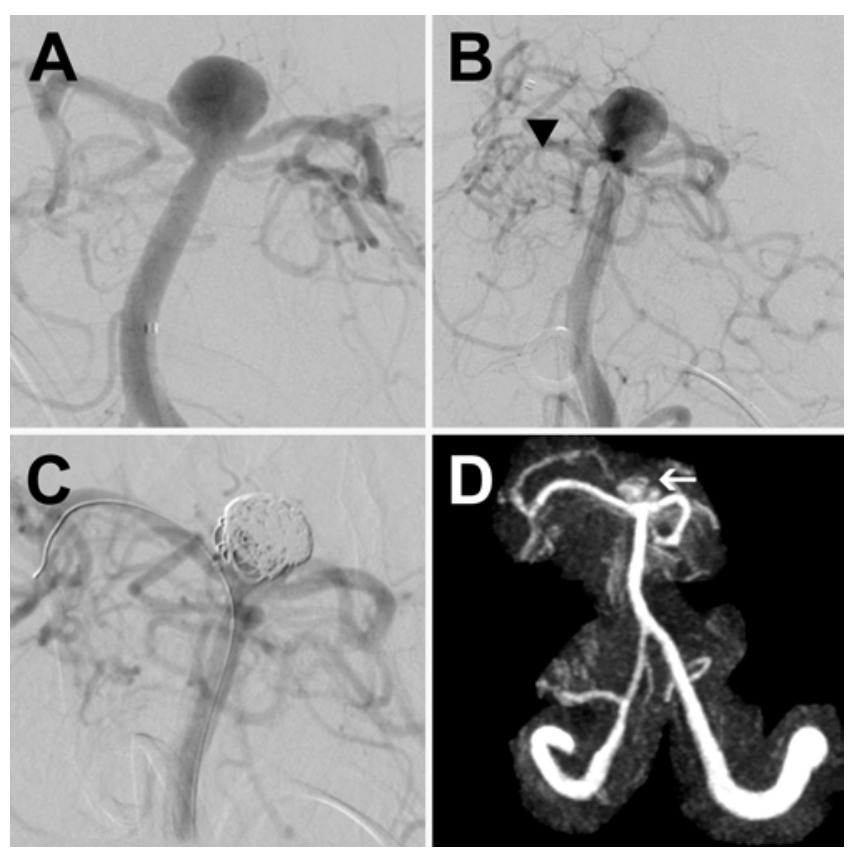

FIG. 4. A: Left vertebral artery angiogram (frontal projection) showing a basilar tip aneurysm targeted for treatment with eCLIPs. B and C: Left vertebral angiogram (frontal projection) obtained after eCLIPs microcatheter delivery into the right PCA (arrowhead, B). Note lack of filling into the right PCA, after which further attempts at eCLIPs delivery were abandoned and the patient was treated with balloon-assisted coiling, resulting in a neck remnant (C). D: Frontal projection of a maximumintensity projection Gd-enhanced MR angiogram obtained 2 months later, demonstrating a large aneurysmal recurrence (arrow). coiling using a Leo stent (Balt Extrusion). The patient had a transient left transient ischemic attack in the MCA distribution, from which he fully recovered. The seventh patient had an unruptured right MCA bifurcation aneurysm; however, after the device was torqued into the correct rotational orientation it could not be unsheathed, and hence unassisted coiling with a loose pack was performed. The eighth patient had an unruptured right ICA terminus aneurysm in which the eCLIPs device was over-torqued in an attempt to obtain the right rotational orientation, and it could not be deployed. Here too the device was removed, and unassisted coiling with a loose pack was achieved.

Acute complications included 2 periprocedural transient ischemic attacks (Cases 22 and 25) and 2 asymptomatic thrombotic events (Case 16 and nondeployed eCLIPs in the first case described above). The first transient ischemic attack occurred during treatment of a 3.5$\mathrm{mm}$ ICA terminus aneurysm, resulting in transient aphasia, which fully recovered. The second was for treatment of a 5.5-mm basilar artery aneurysm and resulted in transient cortical blindness. The first of the asymptomatic events was suspected to be due to antiplatelet therapy resistance, which resolved spontaneously with removal of the device. Transient cortical blindness occurred again during subsequent attempted stent-assisted coiling with a Solitaire AB device (Medtronic). The second involved platelet aggregation of the thrombus after eCLIPs deployment, requiring microcatheter-targeted abciximab administration. Four episodes of significant procedural vasospasm occurred; 2 of these were related to placement of the eCLIPs microcatheter, and 1 case resulted in the decision not to deploy the device. One dissection (Case 8) occurred with initial 4-Fr diagnostic catheterization requiring stenting. No periprocedural deaths were recorded.

Complete data (including that at the time of the procedure and follow-up) were recorded for 21 (84\%) of 25 patients at an average of 8 months (range 3-18 months; Fig. 5). Overall, $9(42.9 \%)$ of these 21 patients demonstrated an improvement in MRRC class at follow-up; no cases of worsening MRRC class were recorded, and $17(81.0 \%)$ of 21 patients sustained a Class I or II angiographic result at follow-up (Fig. 6).

Two aneurysm-related late deaths were noted. Both occurred in patients who had previously undergone coil treatment of symptomatic giant aneurysms, both of which demonstrated increasing mass effect in a fashion similar to that previously described after flow diversion. ${ }^{9}$ In the first case, a $25-\mathrm{mm}$ aneurysm that was initially treated in 2007 with coils presented again with a major recurrence, resulting in subarachnoid hemorrhage. At that time, the aneurysm underwent re-coiling. The patient returned 7 months later for treatment with the eCLIPs device and additional coils. The final posttreatment MRRC class was IIIb. No flow diversion effect was noted after treatment. However, at the 7-month MR angiography follow-up, severe coil compaction was noted, and the aneurysm was re-treated with re-coiling at 9 months, but this treatment did not prevent late rupture at 10 months. The second previously untreated giant unruptured terminal basilar aneurysm was treated with 2 eCLIPs devices and coiling. Posttreatment MRRC Class II occlusion was achieved, with 


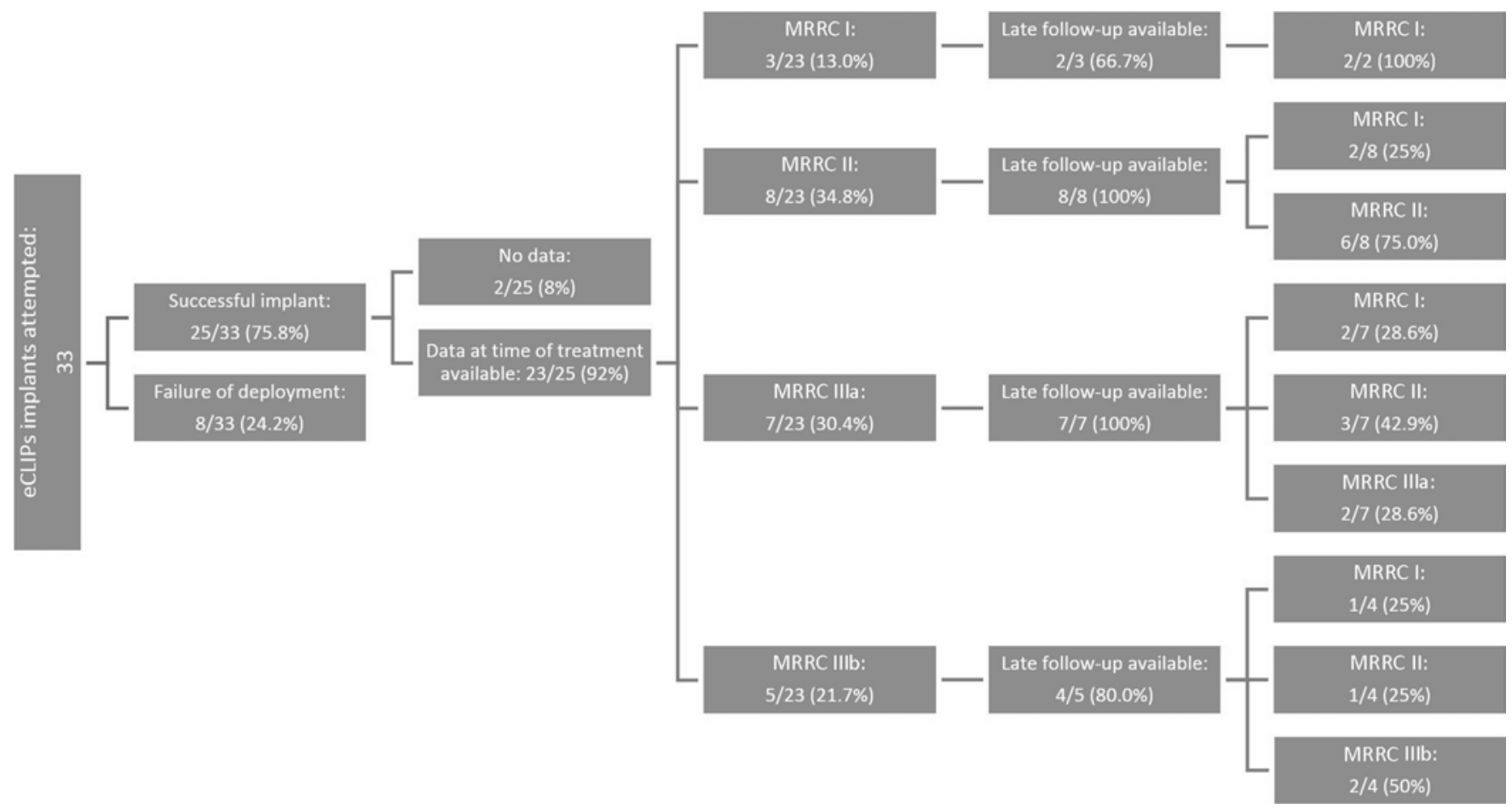

FIG. 5. Flowchart demonstrating the breakdown of data with regard to occlusion according to the MRRC. Raymond Class I, complete obliteration; Class II, residual neck; Class IIla, central interstitial filling; and Class IIIb, interstitial filling adjacent to the aneurysm wall.

mild flow effect noted. Over the next 4 months, however, the patient developed symptoms of increasing mass effect requiring steroid administration. The patient also developed hydrocephalus, requiring CSF diversion 4 months after treatment. Aneurysm enlargement continued, and the patient developed an intraventricular hemorrhage and subsequently died at 4 months. An additional patient died of presumed unrelated causes and had no follow-up, but the initial posttreatment MRRC class was I.

Two re-treatments were attempted. The first is detailed above. The second was performed for an initially $12-\mathrm{mm}$ ruptured aneurysm on Day 2 after subarachnoid hemorrhage with 1 eCLIPs device and coils. An initial MRRC Class IIIa result was obtained. After management for cerebral vasospasm, the patient was sent to a rehabilitation facility, with an outcome modified Rankin Scale score of 0 . However, on follow-up the remnant slowly enlarged, and the patient underwent re-treatment at 20 months without complication. No eCLIPs device migration or displacements during retreatment were noted.

\section{Discussion}

In this multicenter cohort covering 13 centers in North America and Europe, we describe our experience treating wide-necked bifurcation intracranial aneurysms using the second generation of the eCLIPs device. There are currently no licensed devices available in Canada, the US, or Europe for the endoluminal treatment of bifurcation intracranial aneurysms. We developed an endovascular device specifically intended for use in conjunction with embolic coils for the treatment of intracranial aneurysms arising at bifurcations. The Endovascular Clip System (eCLIPs) products were designed to address an unmet clinical need for the treatment of these specific aneurysms.

Treatment of wide-necked intracranial aneurysms is associated with higher recanalization and higher complication rates. ${ }^{5,7}$ Conventional balloon-assisted and stent-assisted coiling techniques only partially address this issue in bifurcation aneurysms, especially if the neck is equally distributed across both branches. Y-stenting is a technique that has been developed to address this issue and was first described in $2004 ;^{2}$ however, this technique is time consuming, and debate has surrounded the potential for higher rates of complications,,${ }^{15}$ likely reflective of the increased requirements for intracranial maneuvers.

The eCLIPs device is specifically designed to address the difficulties of treating wide-necked aneurysms located in anatomical bifurcations. As it is not circumferential in its design, there is no risk of "jailing" out the parent vessel (as in "barrel" stenting) or either daughter or branch vessel (as with either stent-assisted coiling or with other bifurcation-specific assist devices, such as the pCONus Bifurcation Aneurysm Implant [phenox] ${ }^{1,4}$ ). The device, allowing for metal coverage in the range of $23 \%-42 \%$ over the neck, may also theoretically allow for flow diversion and less risk of coil compaction, although this has yet to be borne out in longer-term data; however, early closure data suggest a similar aneurysm closure rate compared with other bifurcation specific devices., ${ }^{1,4}$ The device, being in essence similar to a "hemi-stent," is also more similar to the proven stent-assisted coiling treatment of aneurysms compared with intraaneurysmal devices such as the WEB, 


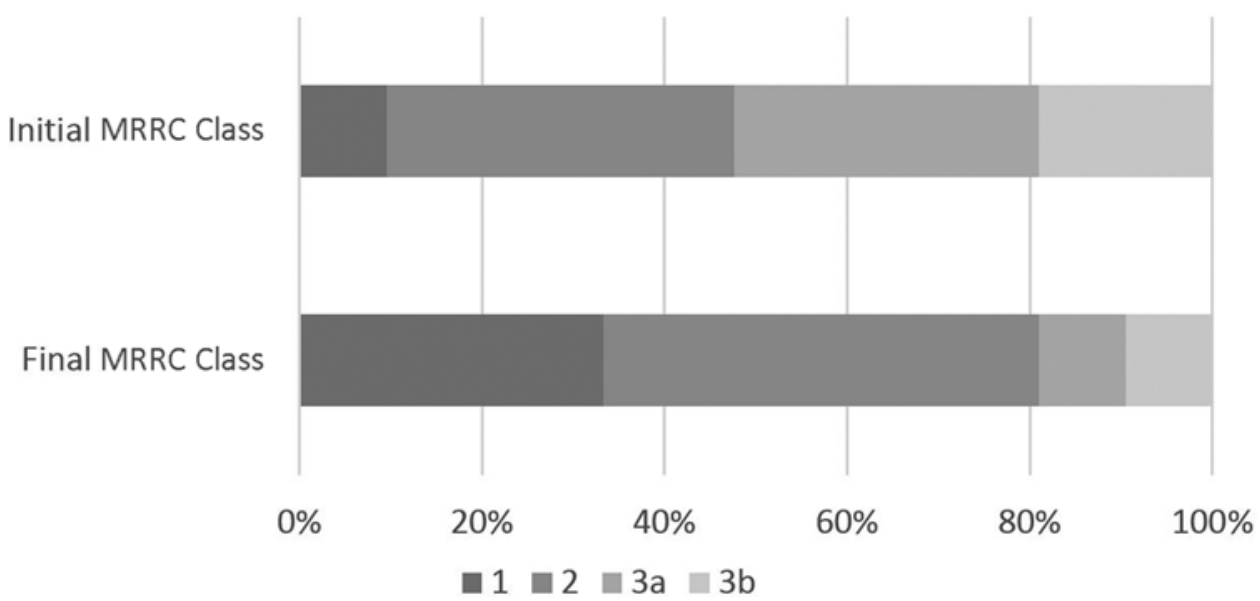

FIG. 6. Bar graph demonstrating the initial and follow-up MRRC classes for all treated aneurysms with complete initial and followup data $(n=21)$.

which also appears to demonstrate similar occlusion rates. ${ }^{3,14}$ Follow-up after eCLIPs implantation, however, revealed no cases of worsening Raymond grade, which is a well-documented issue following stent-assisted coiling (Fig. 7). ${ }^{6}$ This could reflect the flow-disrupting effect of the eCLIPs device.

As with any new investigational device, we acknowledge a learning curve as evidenced by the greater number of failed deployments early in our experience. This is true in regard to not only delivery technique but also case selection. In addition, the deployment technique to achieve correct rotational orientation is different from other neurointerventional devices. The surgeon's familiarity with the device quickly translates into safe, accurate, and reproducible deployment, as was demonstrated in the case registry.

The limitations of this study include its small size and nonrandomized nature, resulting in an inability to allow direct comparisons to other studies for established methods of treating wide-necked aneurysms. As with any new device, greater patient numbers and longitudinal followup are required to directly compare the procedural safety, efficacy, and long-term outcome of this new treatment method. A multicenter, open-label, single-arm feasibility, safety, and efficacy study (the European eCLIPs Safety, Feasibility and Efficacy Study [EESIS]) is underway.

\section{Conclusions}

The second-generation eCLIPs device appears to be a viable option for treatment of both ruptured and unruptured wide-necked bifurcation aneurysms. It is a unique and innovative device, both in design and delivery and, when appropriate case selection is made, allows for better neck coverage facilitating aneurysm obliteration. The device's unique design does not cause jailing of either the parent or branch vessels and allows adjunctive coiling into the aneurysm dome. When compared with pilot data for other bifurcation support devices, the aneurysm occlusion rate in this initial clinical series appears to be favorable, which may reflect the leaf segment's capacity to serve as flow diverter and a platform for neointimal growth over the aneurysm neck.

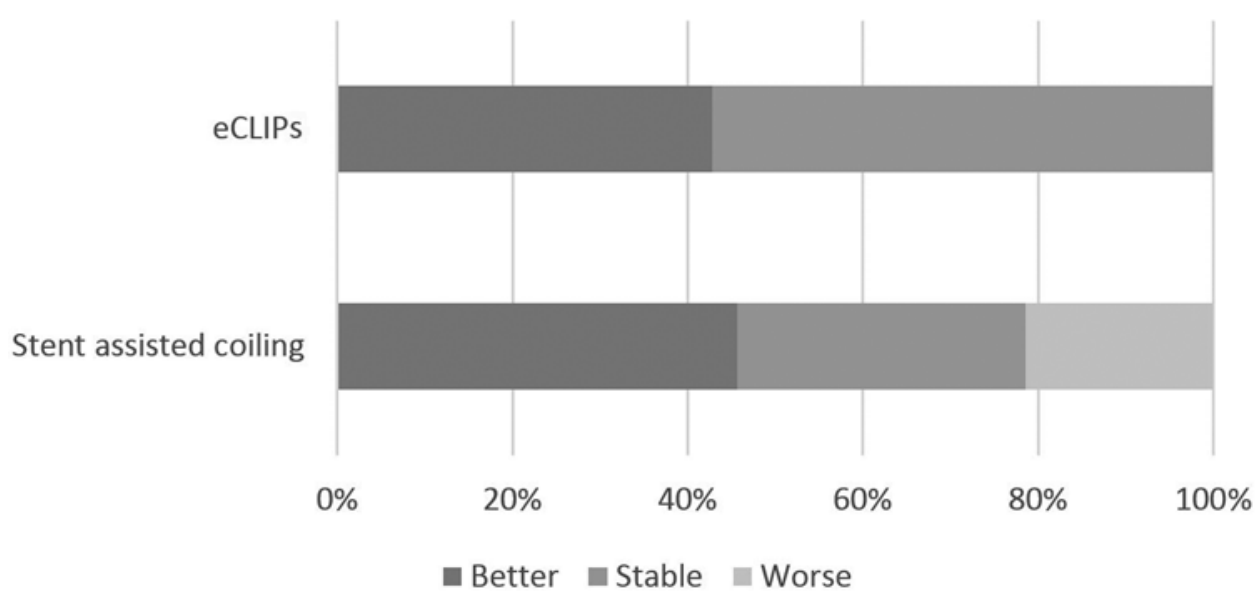

FIG. 7. Bar graph demonstrating the breakdown of changes in MRRC class between time of treatment and follow-up, comparing the eCLIPs device and stent-assisted coiling (data from the study by Hetts et al.). Worsening of MRRC class occurred in more than $20 \%$ of cases treated with stent-assisted coiling, whereas aneurysm filling remained stable or improved with the eCLIPs device. 


\section{Acknowledgments}

We acknowledge Dr. Monika Killer-Oberpfalzer, Christian Doppler Klinik, Forschungsinstitut Für Neurointervention, for providing electron microscopy images.

\section{References}

1. Aguilar-Pérez M, Kurre W, Fischer S, Bäzner H, Henkes H: Coil occlusion of wide-neck bifurcation aneurysms assisted by a novel intra- to extra-aneurysmatic neck-bridging device (pCONus): initial experience. AJNR Am J Neuroradiol 35:965-971, 2014

2. Chow MM, Woo HH, Masaryk TJ, Rasmussen PA: A novel endovascular treatment of a wide-necked basilar apex aneurysm by using a Y-configuration, double-stent technique. AJNR Am J Neuroradiol 25:509-512, 2004

3. Cognard C, Januel AC: Remnants and recurrences after the use of the WEB intrasaccular device in large-neck bifurcation aneurysms. Neurosurgery 76:522-530, 2015

4. Gory B, Aguilar-Pérez M, Pomero E, Turjman F, Weber W, Fischer S, et al: pCONus device for the endovascular treatment of wide-neck middle cerebral artery aneurysms. AJNR Am J Neuroradiol 36:1735-1740, 2015

5. Henkes H, Fischer S, Weber W, Miloslavski E, Felber S, Brew S, et al: Endovascular coil occlusion of 1811 intracranial aneurysms: early angiographic and clinical results. Neurosurgery 54:268-285, 2004

6. Hetts SW, Turk A, English JD, Dowd CF, Mocco J, Prestigiacomo C, et al: Stent-assisted coiling versus coiling alone in unruptured intracranial aneurysms in the matrix and platinum science trial: safety, efficacy, and mid-term outcomes. AJNR Am J Neuroradiol 35:698-705, 2014

7. Hope JK, Byrne JV, Molyneux AJ: Factors influencing successful angiographic occlusion of aneurysms treated by coil embolization. AJNR Am J Neuroradiol 20:391-399, 1999

8. Jahshan S, Abla AA, Natarajan SK, Drummond PS, Kan P, Karmon Y, et al: Results of stent-assisted vs non-stent-assisted endovascular therapies in 489 cerebral aneurysms: singlecenter experience. Neurosurgery 72:232-239, 2013

9. Kulcsár Z, Houdart E, Bonafé A, Parker G, Millar J, Goddard AJ, et al: Intra-aneurysmal thrombosis as a possible cause of delayed aneurysm rupture after flow-diversion treatment. AJNR Am J Neuroradiol 32:20-25, 2011

10. Marotta TR, Gunnarsson T, Penn I, Ricci DR, McDougall I, Marko A, et al: A novel endovascular clip system for the treatment of intracranial aneurysms: technology, concept, and initial experimental results. Laboratory investigation. J Neurosurg 108:1230-1240, 2008

11. Marotta TR, Riina HA, McDougall I, Ricci DR, Killer-Oberpfalzer M: Physiological remodeling of bifurcation aneurysms: preclinical results of the eCLIPs device. J Neurosurg [epub ahead of print March 17, 2017. DOI: 10.3171/2016.10. JNS162024]

12. Mascitelli JR, Moyle H, Oermann EK, Polykarpou MF, Patel AA, Doshi AH, et al: An update to the Raymond-Roy Occlusion Classification of intracranial aneurysms treated with coil embolization. J Neurointerv Surg 7:496-502, 2015
13. Pierot L, Cognard C, Spelle L, Moret J: Safety and efficacy of balloon remodeling technique during endovascular treatment of intracranial aneurysms: critical review of the literature. AJNR Am J Neuroradiol 33:12-15, 2012

14. Pierot L, Liebig T, Sychra V, Kadziolka K, Dorn F, Strasilla $\mathrm{C}$, et al: Intrasaccular flow-disruption treatment of intracranial aneurysms: preliminary results of a multicenter clinical study. AJNR Am J Neuroradiol 33:1232-1238, 2012

15. Spiotta AM, Lena J, Chaudry MI, Turner RD, Turk AS: Ystenting for bifurcation aneurysm coil embolization: what is the risk? Stroke Res Treat 2014:762389, 2014

16. Yang H, Sun Y, Jiang Y, Lv X, Zhao Y, Li Y, et al: Comparison of stent-assisted coiling vs coiling alone in 563 intracranial aneurysms: safety and efficacy at a high-volume center. Neurosurgery 77:241-247, 2015

\section{Disclosures}

The authors report the following. Dr. De Vries: consultant for Evasc; and principal investigator for the EESIS trial, a multicenter postmarketing study evaluating the safety, technical feasibility, and efficacy of the eCLIPs family of products for the treatment of bifurcation intracranial aneurysms, an industry sponsored trial (Evasc). Dr. O'Kelly: proctor for Pipeline Embolization Device insertion for Medtronic. Dr. Riina: medical advisor to and financial stakeholder of Evasc Medical Systems. Mr. McDougall: ownership in, consultant for, and employed by Evasc Medical Systems. Mr. Tippett: employed by Evasc Medical Systems. Ms. Wan: employed by Evasc Medical Systems. Dr. Marotta: direct stock ownership in Evasc, and medical consultant and proctor for eCLIPs, part of Evasc.

\section{Author Contributions}

Conception and design: Marotta. Acquisition of data: Marotta, Chiu, De Vries, O'Kelly. Analysis and interpretation of data: Marotta, Chiu, O'Kelly. Drafting the article: Marotta, Chiu. Critically revising the article: Marotta, Chiu, De Vries, O'Kelly, Riina, de Oliveira Manoel. Reviewed submitted version of manuscript: Marotta, Chiu, De Vries, O'Kelly, Riina, McDougall, Tippett, Wan. Approved the final version of the manuscript on behalf of all authors: Marotta. Administrative/technical/material support: McDougall, Tippett, Wan.

\section{Supplemental Information Videos \\ Video 1. https://vimeo.com/191976808.}

\section{Previous Presentations}

Partial data were presented at the 13th Congress of the World Federation of Therapeutic and Interventional Neuroradiology in Gold Coast, Australia.

\section{Correspondence}

Thomas R. Marotta, Diagnostic and Therapeutic Neuroradiology, St. Michael's Hospital, 30 Bond St., Toronto, ON M5B 1W8, Canada. email: marottat@smh.ca. 